\title{
Modeling ion effects for the Argonne Advanced Photon Source upgrade
}

\author{
J. Calvey ${ }^{*}$ and M. Borland \\ Advanced Photon Source, Argonne National Laboratory, 9700 South Cass Avenue, \\ Argonne, Illinois 60439, USA
}

(Received 3 May 2019; published 15 November 2019)

\begin{abstract}
Ions are produced in an accelerator when the beam ionizes residual gas inside the vacuum chamber. If the beam is negatively charged, ions can become trapped in the beams potential, and their density will increase over time. Trapped ions can cause a variety of undesirable effects, including instability and emittance growth. This paper will discuss the possibility of ion trapping and instability in the APS-Upgrade storage ring. The question of where ions will be trapped is addressed using simple analytical calculations, while the instability is modeled with computer simulations. A scheme is proposed for mitigating the ion instability, by using train gaps with a high charge bunch before and after the gap to minimize rf transients. The reduction in ion density due to nonevaporable getter coating is also discussed.
\end{abstract}

DOI: $10.1103 /$ PhysRevAccelBeams.22.114403

\section{INTRODUCTION}

The APS-Upgrade is a 4th-generation light source currently under development at Argonne National Laboratory [1]. The lattice design [2] utilizes a seven-bend hybrid multibend achromat [3] (hybrid MBA) with reverse bending magnets $[4,5]$ to achieve a natural emittance of $42 \mathrm{pm}$ at $6 \mathrm{GeV}$. The lattice functions for one sector of the APS-U storage ring are shown in Fig. 1. The hybrid MBA cell is symmetric and features two dispersion bumps, within each of which are placed three sextupoles. As indicated in the figure, these are referred to as the "multiplet" sections, since they contain quadrupoles, sextupoles, skew quadrupoles, and octupoles. These sections also have larger-than-average beta functions, which has implications for ion trapping, as will be seen below.

Some basic parameters of the lattice are given in Table I. There are several modes of operation planned, including a 48-bunch timing mode and a 324-bunch brightness mode.

Because of the challenging emittance and stability requirements of the APS-U, ion trapping is a serious concern. Trapped ions can lead to quickly growing transverse (usually vertical) instability, due to coupled motion between the beam and the ions. The strength of the instability is generally proportional to the average beam current, and inversely proportional to the beam size [6]. Thus ions are particularly dangerous for the APS-U, which will have high current and low emittance.

\footnotetext{
jicalvey@anl.gov
}

Published by the American Physical Society under the terms of the Creative Commons Attribution 4.0 International license. Further distribution of this work must maintain attribution to the author(s) and the published article's title, journal citation, and DOI.
However, very high beam densities can actually overfocus the ions, preventing them from being trapped in the first place. The question of which parts of the APS-U lattice will trap ions is discussed in Sec. II. This can provide guidance on the vacuum design, for example in deciding where NEG coating is required.

Ion instability is best studied quantitatively using computer simulations (Sec. III). For the APS-U storage ring, the simulations indicate that train gaps are required to prevent the instability. In Sec. IV, we propose using high charge "guard bunches" before and after the train gaps, to minimize rf transients and provide a stronger kick for clearing out ions.

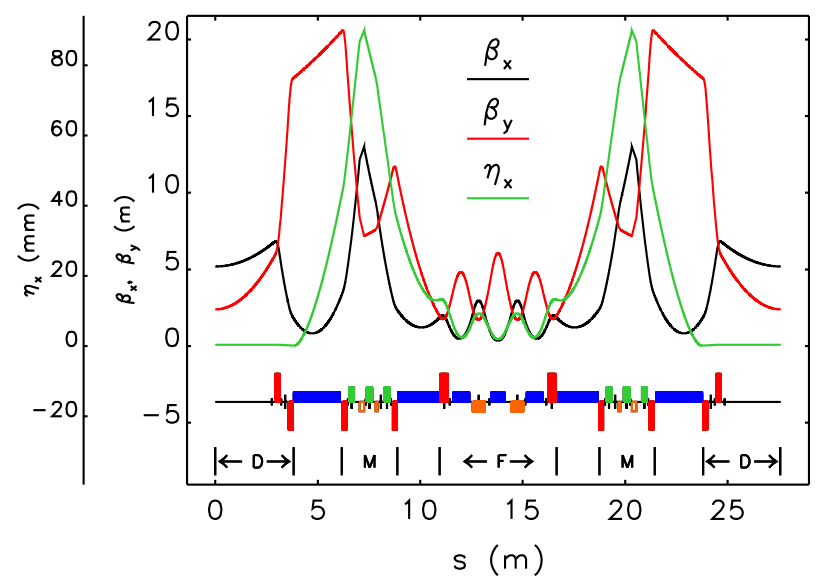

FIG. 1. Lattice functions for one sector of the APS-U storage ring, broken into doublet (D), multiplet (M), and FODO (F) sections. Blue objects are normal-direction dipoles, orange objects are reverse-direction dipoles, red objects are quadrupoles, and green objects are sextupoles. Skew quadrupole and (potential) octupole magnets are incorporated into eight-pole magnets in the doublet and multiplet sections, but these are not shown. 
TABLE I. APS-U storage ring parameters for two modes of operation.

\begin{tabular}{|c|c|c|}
\hline Quantity & & Value \\
\hline Beam energy & & $6 \mathrm{GeV}$ \\
\hline Natural emittance & & $42 \mathrm{pm}$ \\
\hline Circumference & & $1104 \mathrm{~m}$ \\
\hline Revolution time & & $3.68 \mu \mathrm{s}$ \\
\hline Beam current & & $200 \mathrm{~mA}$ \\
\hline Quantity & Timing & Brightness \\
\hline Bunches & 48 & 324 \\
\hline Bunch spacing & $77 \mathrm{~ns}$ & $11 \mathrm{~ns}$ \\
\hline Bunch charge & $15.4 \mathrm{nC}$ & $2.2 \mathrm{nC}$ \\
\hline
\end{tabular}

\section{TRAPPING CRITERIA}

For a machine without an ion-clearing train gap [7], Bacconier and Brianti [8] derived a simple expression for determining whether a given ion will be trapped by the beam (see Appendix for details). The trapping can be characterized by a local critical mass number given by

$$
A_{x, y}=\frac{N_{e} r_{p} S_{b} Q}{2 \sigma_{x, y}\left(\sigma_{x}+\sigma_{y}\right)},
$$

where $N_{e}$ is the bunch population, $r_{p} \approx 1.5 \times 10^{-18} \mathrm{~m}$ is the classical proton radius, $S_{b}$ is the bunch spacing, $\sigma_{x}$ and $\sigma_{y}$ are the horizontal and vertical beam sizes, and $Q$ is the charge number of the ion $(=1$ for a singly ionized molecule). Ions with mass number larger than $A_{q}$ will be trapped in plane $q$, in that their motion in plane $q$ will be stable under the influence of the field of the electron beam; lighter ions will not be trapped. Ions are only trapped (i.e., trapped in both planes) if they have atomic number $A>A_{\text {crit }} \equiv \max \left(A_{x}, A_{y}\right)$. In 48-bunch mode $\left(N_{e}=9.6 \times 10^{10}, S_{b}=23 \mathrm{~m}\right), A_{\text {crit }}>700$ for the entire ring, so ions will definitely not be trapped, and ion instability should not be an issue. Hence, all results presented in this paper are for 324-bunch mode $\left(N_{e}=\right.$ $1.4 \times 10^{10}, S_{b}=3.4 \mathrm{~m}$ ).

The critical mass will vary around the ring (since it depends on the beam size), so a given ion may be trapped in some parts of a lattice, but not others. This is depicted graphically in Fig. 2, which compares the trapping parameters for the APS-U lattice to the mass numbers of different gasses. The plot shows that trapping occurs primarily in the multiplet sections, where the beta functions and dispersion are large (see Fig. 1). It is also clear that round beamsthose for which both the horizontal and vertical beam size are large-will trap more ions than flat beams.

Table II gives the fraction of the $42 \mathrm{pm}$ APS-U lattice that will trap singly-ionized $\mathrm{H}_{2}(A=2), \mathrm{CH}_{4}(A=16)$, $\mathrm{CO}(A=28)$, and $\mathrm{CO}_{2}(A=44)$ ions. Results are given for four different emittance ratios. With a relatively flat beam

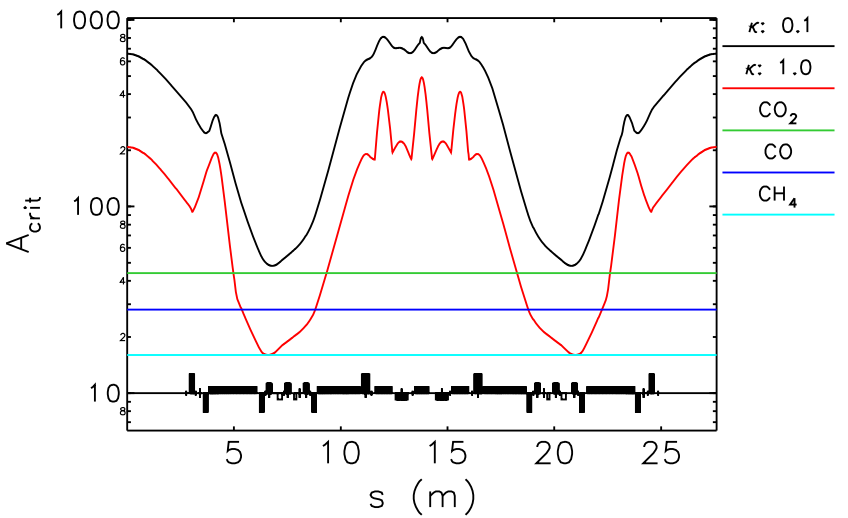

FIG. 2. Critical mass for different emittance ratios $(\kappa)$, compared with the mass numbers of common gasses. An ion will be trapped wherever its mass number is larger than the critical mass.

$\left(\kappa \equiv \varepsilon_{y} / \varepsilon_{x}=0.1\right)$, no ion trapping is expected. With rounder beams $(\kappa>0.1)$, heavier ions will be trapped in a portion of the lattice. In order to maximize the Touschek lifetime [9], operation with $\kappa \approx 1$ is planned for APS-U [10], though this is less critical for 324-bunch mode.

\section{A. Ion frequency}

Ions at a given location in the lattice will oscillate around the beam with a characteristic frequency given by Eq. (2). Ion instability occurs when the motion of the ions couples to a beam mode, leading to exponential growth of the beam motion. The instability manifests as elevated lower betatron sidebands near the ion frequency.

$$
\omega_{i, y} \approx c\left(\frac{4 N_{e} r_{p} Q}{3 A S_{b}\left(\sigma_{x}+\sigma_{y}\right) \sigma_{y}}\right)^{1 / 2} .
$$

The numerical factor inside the square root comes from integration over the beam and ion distributions [11]. Note that by combining Eqs. (1) and (2), one can find an alternate way of expressing the trapping condition:

$$
f_{b}>\sqrt{\frac{3}{2}} \pi f_{i}
$$

where $f_{i}=\omega_{i} /(2 \pi)$ and $f_{b}$ is the bunch frequency $\left(c / S_{b}\right)$. Qualitatively, this means the bunch frequency must be much larger than the ion frequency for trapping to occur. In this case, the beam appears quasicontinuous to the ions.

TABLE II. Percent of lattice that will trap ions in 324-bunch mode, for different emittance ratios.

\begin{tabular}{lcccccc}
\hline \hline$\kappa$ & $\varepsilon_{x}(\mathrm{pm})$ & $\varepsilon_{y}(\mathrm{pm})$ & $\% \mathrm{H}_{2}$ & $\% \mathrm{CH}_{4}$ & $\% \mathrm{CO}$ & $\% \mathrm{CO}_{2}$ \\
\hline 0.1 & 40 & 4 & 0 & 0 & 0 & 0 \\
0.2 & 39 & 8 & 0 & 0 & 0 & 15 \\
0.4 & 36 & 14 & 0 & 0 & 10 & 25 \\
1.0 & 29 & 29 & 0 & 0 & 25 & 32 \\
\hline \hline
\end{tabular}




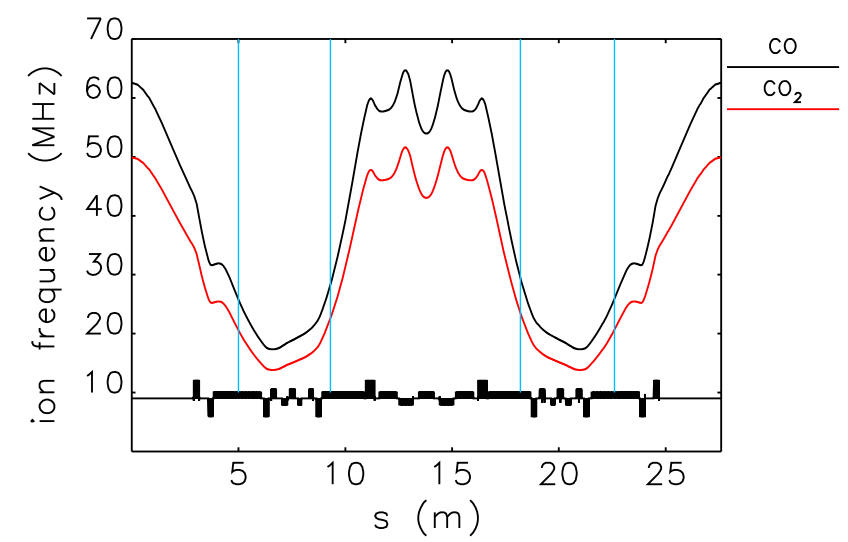

FIG. 3. Ion frequencies for one sector of the APS-U lattice, 324 bunches, round beams, $200 \mathrm{~mA}$. The vertical lines indicate the regions where $\mathrm{CO}_{2}$ ions are trapped (the $\mathrm{CO}$ trapping regions are slightly narrower).

Like the critical mass, the ion frequency will vary along the lattice. Figure 3 shows the ion frequency for $\mathrm{CO}$ and $\mathrm{CO}_{2}$ along one sector of the APS-U lattice, for 324 bunch mode. Of course this is most relevant where the ions are actually trapped, i.e., in the multiplet sections. For this region the characteristic frequency is between 14 and $23 \mathrm{MHz}$.

\section{INSTABILITY SIMULATIONS}

Ion instability at the APS-U has been investigated using a simulation code developed at SLAC [11,12], which models the interaction between the beam and ions at multiple points around the ring. In this code the ions are modeled using macroparticles, but the beam is rigid, with only centroid motion allowed. The user supplies a lattice file and pressure profile (see below), and the code automatically generates multiple interaction points $(\sim 1400$ in the case of APS-U). For each interaction point and each bunch passage, the code models the generation of ions (based on the local pressure of each gas), the motion of ions during the bunch gap, and the interaction between the beam and ions. The beam is modeled by a single macroparticle with an assumed Gaussian distribution, and the kick to the ions from the beam is calculated using the Bassetti-Erskine formula [13]. The kick to the beam from the ion cloud is calculated using a Poisson solver. The simulations include radiation damping, but not coherent damping or feedback. This code has been benchmarked with ion-induced tune shift measurements in the APS Particle Accumulator Ring [14].

\section{A. Pressure profiles}

The simulations incorporate realistic vacuum profiles, generated using the CERN codes SynRad+ and MolFlow+ $[15,16]$. SynRad+ models the generation and scattering of photons to compute a photon flux distribution on the chamber wall. MolFlow+ uses this as input to model photon stimulated desorption of gas molecules, as well as vacuum

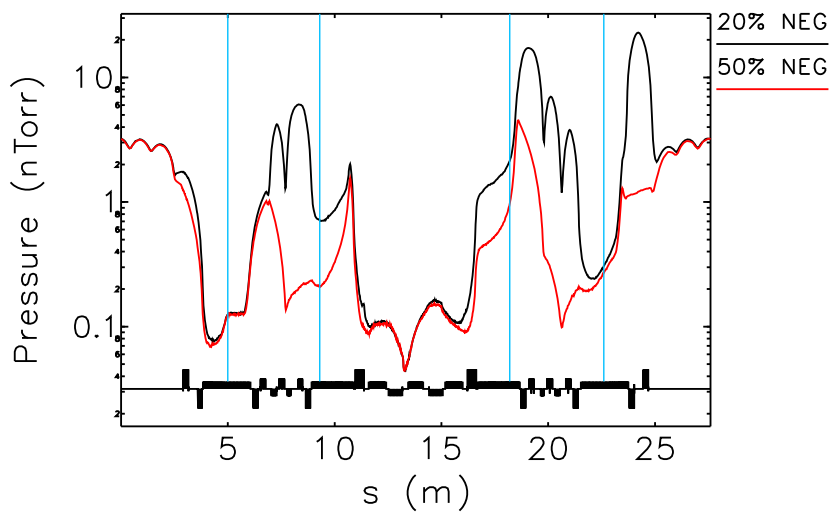

FIG. 4. Pressure profiles for one sector of the APS-U storage ring, calculated by MolFlow+, for 1000 Ah beam scrubbing and $200 \mathrm{~mA}$ beam current. The vertical lines indicate the regions where $\mathrm{CO}_{2}$ ions are trapped.

conductance and pumping. The end result is the partial pressure for different gas molecules around the ring, for a given beam scrubbing time and beam current.

The initial APS-U vacuum design [17] incorporated NEG coating only in the FODO sections, which amounts to $\sim 20 \%$ of the ring. More recently, it was decided to coat the multiplet and doublet sections, so that $\sim 50 \%$ of the ring is coated [18]. Since the multiplet chambers are made of aluminum, they will not be baked at the standard NEG activation temperature of $200^{\circ} \mathrm{C}$, but rather at $150^{\circ} \mathrm{C}$. This is required to preserve the temper and yield strength of aluminum. Therefore the vacuum simulations make the conservative assumption that the NEG coating in the multiplet sections provide no additional pumping. Instead, the benefit comes entirely from the reduction in photon stimulated desorption [19].

Figure 4 shows the total pressure profile for one sector of the ring, for $20 \%$ and $50 \%$ NEG coating. The profiles were generated by MolFlow+, with 1000 Ah beam scrubbing, which corresponds to roughly one year of operation at full current. For 50\% NEG coating, the gas composition is $43 \%$ $\mathrm{H}_{2}, 36 \% \mathrm{CO}, 13 \% \mathrm{CO}_{2}$, and $8 \% \mathrm{CH}_{4}$.

NEG coating reduces the average pressure from $2.75 \mathrm{nTorr}$ to $0.93 \mathrm{nTorr}$. The effect is even more significant in the multiplets, where the ions are trapped (Fig. 2).

The ion simulation code uses the total pressure profile, multiplied by the average gas composition around the ring, to estimate the local partial pressure of each gas. This turns out to be a reasonable approximation; for example, Fig. 5 compares the partial pressure of $\mathrm{CO}$ given by MolFlow+ with the pressure given by this estimate. The agreement is generally quite good.

\section{B. Simulation results}

Figure 6 plots the simulated ion density vs time for round beams $(\kappa=1.0)$ and flat beams $(\kappa=0.1)$, and for $20 \%$ and $50 \%$ NEG coating. As predicted by the calculations in 


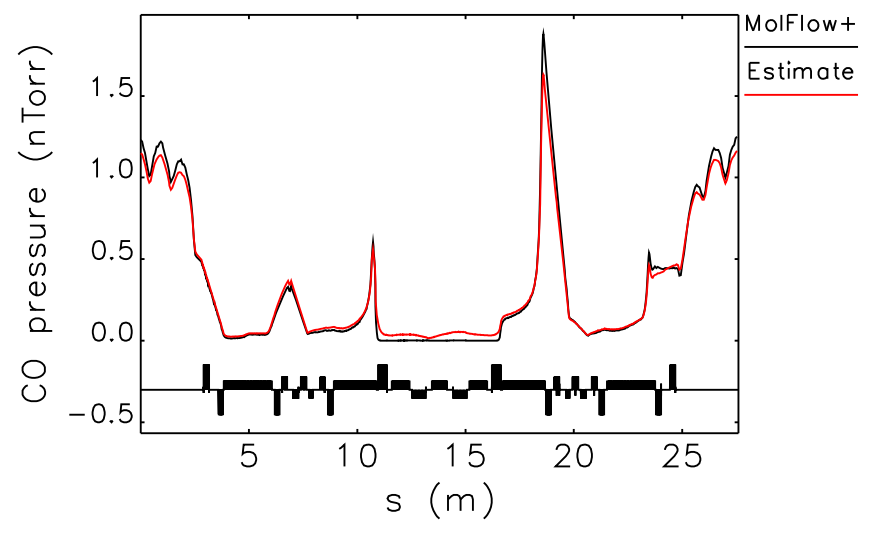

FIG. 5. Comparison of MolFlow+ calculation of the $\mathrm{CO}$ partial pressure with the estimate used by the ion code. The conditions are $1000 \mathrm{Ah}, 200 \mathrm{~mA}$, and 50\% NEG coating.

Sec. II, no trapping is observed for the flat beam case (i.e., the ion density does not increase with time), while ions are trapped in the round beam case. Of course, the density is higher for $20 \%$ NEG than for 50\% NEG.

As shown in Fig. 7, ion trapping in the round beam case leads to a strong vertical instability. The instability amplitude initially grows very quickly, then saturates when the beam motion reaches about $10 \%$ of the vertical beam size, after which it grows much more slowly. Even this small amount of beam motion is enough to shake out some of the ions, leading to a reduction in the ion density (which can be seen clearly in Fig. 6). Thus the instability is to some extent self-limiting, though unfortunately not to the extent that it stops growing altogether.

The self-clearing mechanism is effective because ions are being driven at their oscillation frequency [Eq. (2)]. This frequency also appears in the beam spectrum as elevated lower vertical betatron sidebands. Figure 8 shows the upper and lower sideband amplitudes up to the Nyquist frequency, for 324 bunch mode (with round beams and 50\% NEG coating). There is a broad peak in the lower sidebands. The peak is strongest between

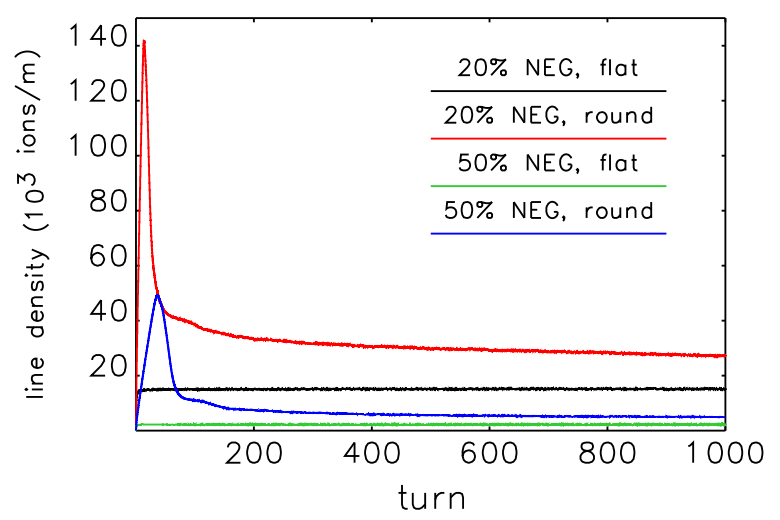

FIG. 6. Simulated ion density (averaged around the ring) for 324 bunches, $200 \mathrm{~mA}, 1000$ Ah conditioning.

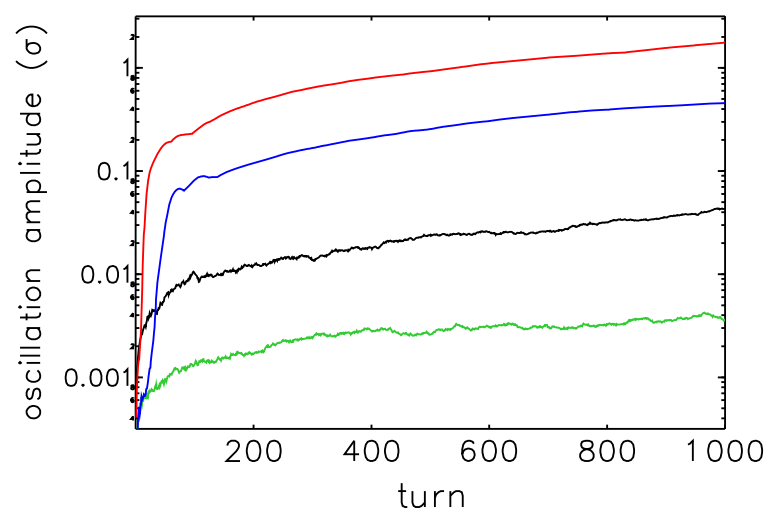

FIG. 7. Ion instability amplitude (in units of vertical beam size) for 324 bunches, $200 \mathrm{~mA}, 1000$ Ah conditioning. Black: 20\% NEG, flat; red: $20 \%$ NEG, round; green: $50 \%$ NEG, flat; blue: $50 \%$ NEG, round.

$\sim 15-24 \mathrm{MHz}$, consistent with the prediction made in Sec. II A. As expected, there is no elevation of the upper sidebands.

The reduction in ion density due to the increased NEG coverage has a strong effect on the ion instability (Fig. 7) the initial instability growth rate (before saturation) is reduced from $\sim 31 / \mathrm{msec}$ to $\sim 15 / \mathrm{msec}$. The final amplitude is also much smaller.

There are three expected sources of vertical damping in the APS-U: synchrotron radiation $(0.062 / \mathrm{msec})$, coherent damping ( $\sim 6.80 / \mathrm{msec}$, though note this is not exponential damping) [20,21], and a transverse feedback system [22] $(\sim 10 / \mathrm{msec})$. Unfortunately, none of these will be fast enough to damp the ion instability, even with 50\% NEG coating.

The flat beam simulations also show an instability, though with a much lower growth rate than the round beam case. However, flat beams are undesirable for beam lifetime, and would be vulnerable to ion induced emittance growth. Use of flat beams would also introduce a complication for x-ray beamline optics, in that 48-bunch mode

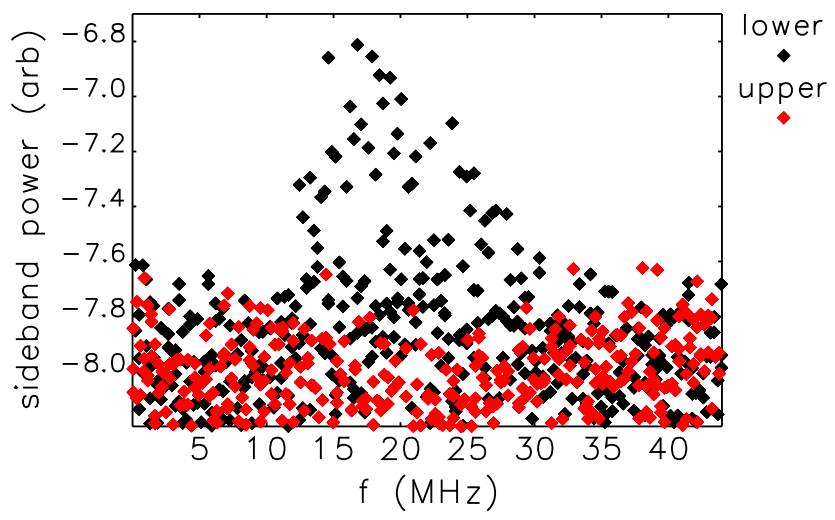

FIG. 8. Vertical betatron sidebands of the beam motion, 324 bunches, round beams, $200 \mathrm{~mA}, 50 \%$ NEG coating. 


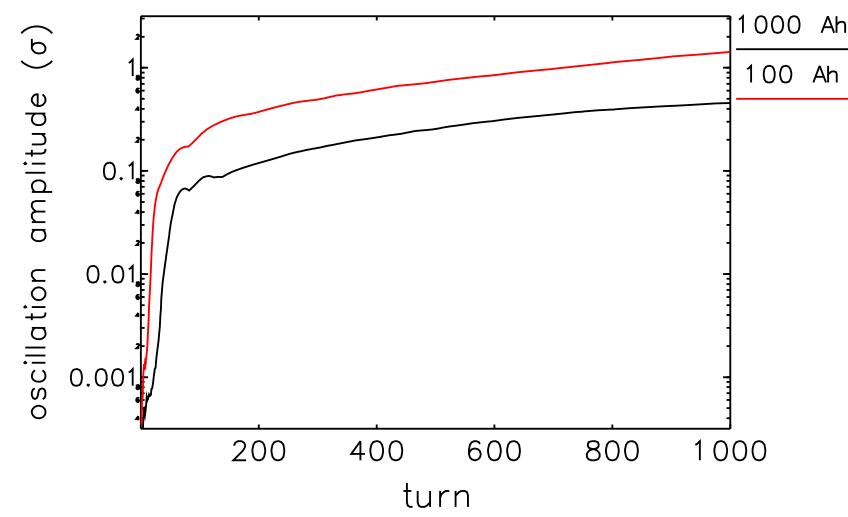

FIG. 9. Ion instability amplitude (in units of vertical beam size) for 324 bunches, $200 \mathrm{~mA}$, comparing $100 \mathrm{Ah}$ and $1000 \mathrm{Ah}$ conditioning.

must use round beams. Therefore we consider another option for mitigating the instability in Sec. IV.

For the rest of this paper, we assume 50\% NEG coating (as planned for the APS-U).

\section{Commissioning and early operation}

In the first year of operation, the vacuum pressure will be higher, and the ion instability will be more severe. The vacuum simulations have also been run for $100 \mathrm{Ah}$ conditioning, which corresponds to early user operation at full current. The average pressure at $100 \mathrm{Ah}$ is significantly higher (3.54 nTorr, compared to 0.93 nTorr for $1000 \mathrm{Ah}$ ). The results are qualitatively similar to the 1000 Ah case, but the ion densities and instability growth rates (Fig. 9) are higher.

Going back even further, it will be desirable to avoid ion instability during commissioning, before full current is reached. From Eq. (1), it is clear that lower bunch charge will lower the critical mass, resulting in more ions being trapped. Rewriting this equation in terms of total beam current $\left(I_{b}\right)$, ring circumference $\left(C_{\text {ring }}\right)$ and the number of bunches $\left(n_{b}\right)$ gives Eq. (4):

$$
A_{x, y}=\frac{I_{\text {beam }} r_{p} C_{\text {ring }}^{2} Q}{2 e c n_{b}^{2} \sigma_{x, y}\left(\sigma_{x}+\sigma_{y}\right)} .
$$

This equation makes explicit that for a given beam current, the critical mass can be raised by using fewer bunches (since this increases the bunch spacing and the bunch charge). For example, consider a point during commissioning where we have $25 \mathrm{~mA}$ total current. Figure 10 plots the critical mass for different $n_{b}$, compared to the mass of $\mathrm{CO}_{2}$. Trapping can be avoided for the whole lattice as long as fewer than 81 bunches are used. This is confirmed by simulations: Fig. 11 shows the simulated instability growth rate as a function of the number of bunches. The growth rate is negligible for up to 72 bunches; at 81 bunches the trapping threshold has been crossed and

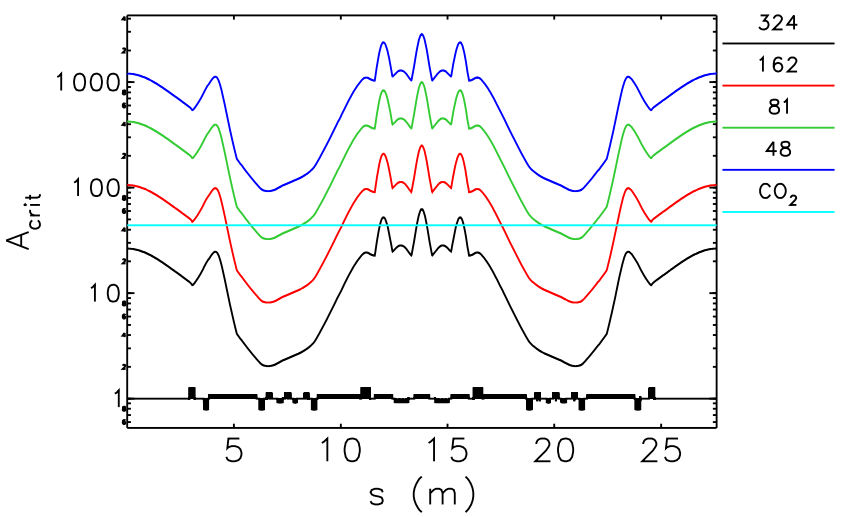

FIG. 10. Critical mass for $25 \mathrm{~mA}$ total beam current. Each line represents the critical mass for a different number of equally spaced bunches.

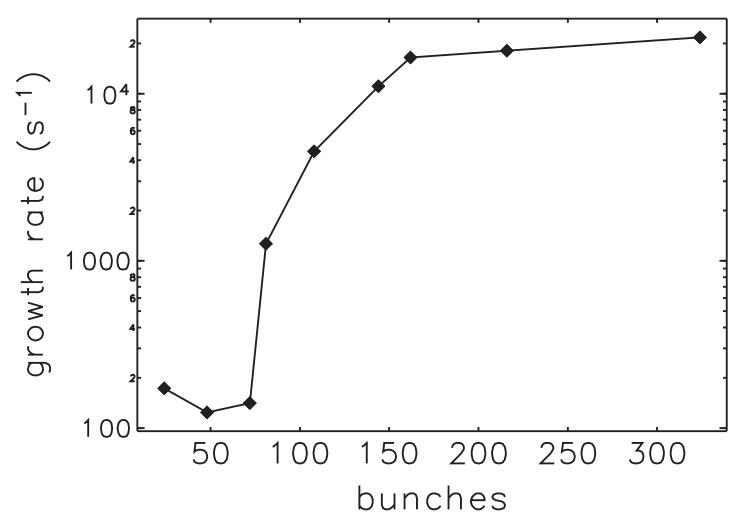

FIG. 11. Instability growth rate as a function of the number of bunches, with $25 \mathrm{~mA}$ total beam current and $5 \mathrm{Ah}$ beam conditioning.

there is a sudden jump in the growth rate. Above the threshold the instability becomes stronger with more bunches, as more and more ions are trapped. The pressure profile used for this simulation assumes $5 \mathrm{Ah}$ conditioning.

\section{COMPENSATED TRAIN GAPS}

One common technique for mitigating ion instability is to use gaps between bunch trains, to allow the ions to clear out [7]. The ion density will still increase along the bunch train, potentially leading to a "fast-ion" instability [23-25], but this is less dangerous than letting the ions accumulate over many turns.

A downside of this technique is that the missing bunches can cause transients in the rf system, leading to variations in the bunch length, phase, and lifetime along the train [26-28]. These effects can be minimized by distributing the missing charge to the bunches adjacent to the gaps, which we refer to as "guard bunches". An example of this scheme is shown in Fig. 12, which depicts four trains with a two bunch gap between them. The guard bunches before and 


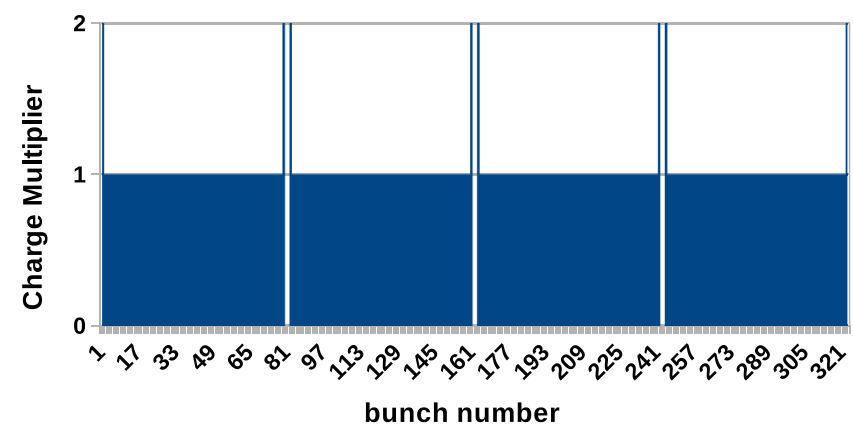

FIG. 12. Bunch population with four compensated gaps. Each gap consists of two empty bunches, with the missing charge distributed to the bunches before and after the gap.

after the gaps have double charge. As discussed below, simulations show that this arrangement should have a modest impact on the longitudinal parameters and Touschek lifetime of the bunches. In addition, the high charge guard bunches before the gaps will provide a stronger kick to the ions, helping with the clearing process. A more quantitative explanation of this effect is given in the Appendix.

\section{A. Impact on bunch properties and beam lifetime}

PELEGANT [29,30], the parallel version of the program ELEGANT [31] was used to model the cases with ordinary and compensated gaps. The program is capable of simultaneously simulating multibunch and single-bunch collective effects in storage rings and linacs [32]. Parallel efficiency is good for up to several hundred cores [33], with scaling limited by the relatively small number of simulation particles (10 to 100's of thousands) needed in such simulations.

Since our primary interest is in the longitudinal beam properties, we did not include any transverse collective effects. Both geometric and resistive components of the longitudinal impedance were included [34] using a single, lumped element. The longitudinal impedance has two main effects. First, it increases the energy spread, due to the microwave instability (MWI), which has a threshold of about $1 \mathrm{~mA}$, just above (below) the current of the main (guard) bunches [35]. Second, it lengthens the bunch, due to potential well distortion. The ordinary and guard bunches are expected to differ somewhat in energy spread and bunch length, if only because of the short-range impedance.

Table III gives parameters of the main and harmonic cavities. The 12 main $352-\mathrm{MHz}$ rf cavities contribute to the longitudinal impedance, but are modeled separately as resonators, including an external generator and its feedback system [36]. For present purposes, the 12 cavities are treated as identical and as if they are driven by a single rf source, though in fact they will be driven by two rf sources. The cavities are detuned to the negative side of the harmonic for Robinson stability and to minimize generator power.
TABLE III. Cavity parameters for APS-U. $R_{a}$ is the shunt impedance using the accelerator definition.

\begin{tabular}{lccccc}
\hline \hline $\begin{array}{l}\text { Cavity } \\
\text { type }\end{array}$ & $\begin{array}{c}\text { Number } \\
\text { present }\end{array}$ & Harmonic & $R_{a} / Q \Omega$ & $\mathrm{Q}$ & $\begin{array}{c}\text { Loaded } \\
\mathrm{Q}\end{array}$ \\
\hline Main & 12 & 1296 & 228 & $48 \times 10^{3}$ & $7.36 \times 10^{3}$ \\
Harmonic & 1 & 5184 & 103 & $2 \times 10^{8}$ & $600 \times 10^{3}$ \\
\hline \hline
\end{tabular}

The 4th-harmonic bunch-lengthening cavity (BLC) [37] is also modeled as a separate resonator, but in this case no $\mathrm{rf}$ generator or feedback system is needed as the cavity is passive. The BLC is detuned to the positive side of the harmonic in order to achieve bunch lengthening and, in particular, to maximize the Touschek lifetime [10]. For the present APS-U lattice and vacuum chamber impedance, detuning by $11 \mathrm{kHz}$ maximizes the Touschek lifetime for a uniform 324-bunch, $200 \mathrm{~mA}$ fill. Hence, the BLC is detuned by $11 \mathrm{kHz}$ for the present simulations. The optimum for the guard bunches would presumably be somewhat closer to resonance, given that the optimum for a uniform 48-bunch fill is $9 \mathrm{kHz}$.

The storage ring lattice is modeled as using a single ILMATRIX (Individualized Linear Matrix) element, which allows simulating a storage ring by computing a linear matrix for each simulation particle on each turn. The matrix is constructed from knowledge of the lattice functions and tunes, plus chromatic derivatives of the lattice functions (up to linear order), the chromaticities (up to third order), the tune shifts with amplitude (up to second order), the momentum compaction (up to second order), and the amplitude-dependence of the path length (up to second order). Hence, considerable relevant detail is included in the beam dynamics without the time penalty associated with element-by-element tracking or spurious effects associated with higher-order matrix formalisms. Synchrotron radiation is also included in a lumped fashion, applied once per turn.

It is assumed that the fill is perfect, i.e., that all the main bunches have the same charge $Q_{m}$ and all the guard bunches have charge $2 Q_{m}$. The charge per bunch is below the MWI threshold, so a reliable simulation of single-bunch effects can be obtained with 10000 simulation particles per bunch. Scans show that this number of particles provides reliable simulation of these higher-charge bunches.

Once the beam has equilibrated (about 30000 turns), slice analysis is used to record emittance, energy spread, and local charge density for each bunch in 30 slices.

Once these simulations have completed, slice-based computation of the Touschek lifetime is performed [10] for each bunch, using 100 instances of slice analysis data for each bunch, taken at ten-turn intervals. For each bunch, the 100 resulting lifetime values are averaged to get the final predicted lifetime value for that bunch. Figures 13-16 show the charge distribution for bunch 0 (normal charge), 


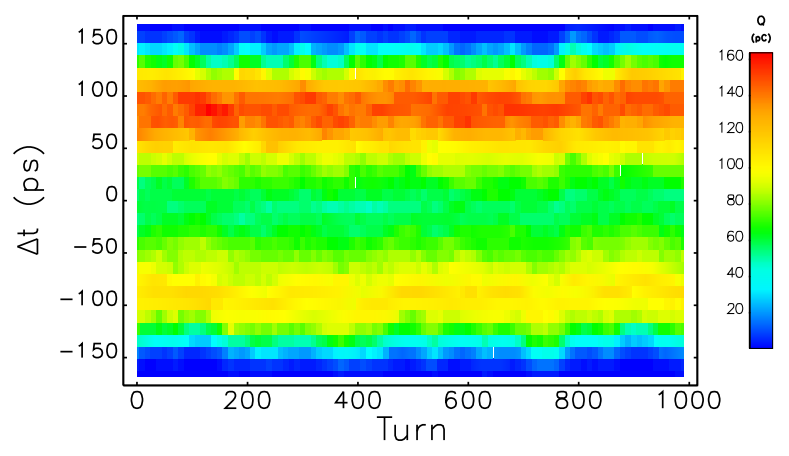

FIG. 13. Charge distribution over 1000 turns from 30-slice analysis for ordinary bunch 0 of a fill with twelve 2-bunch gaps.

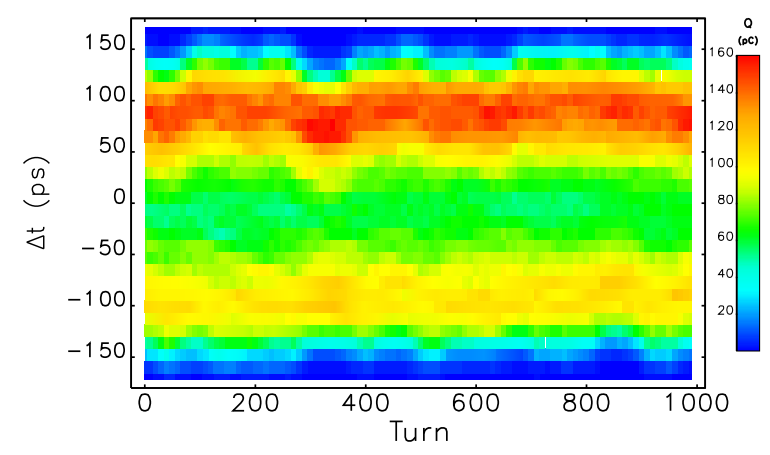

FIG. 14. Charge distribution over 1000 turns from 30-slice analysis for ordinary bunch 22 of a fill with twelve 2-bunch gaps.

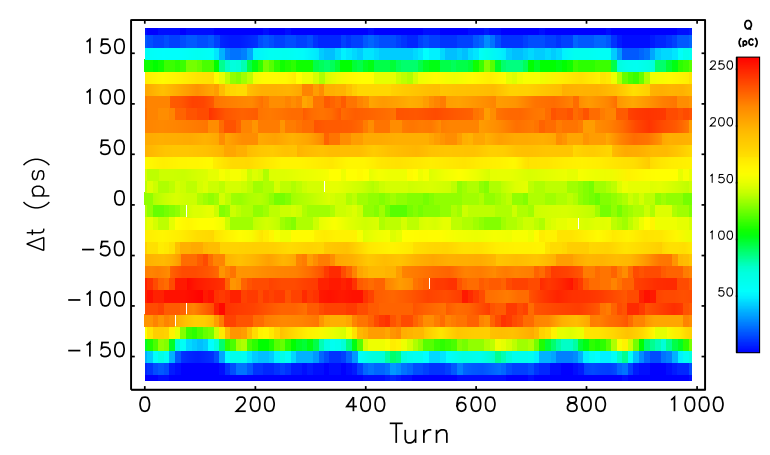

FIG. 15. Charge distribution over 1000 turns from 30-slice analysis for guard bunch 23 of a fill with twelve 2-bunch gaps.

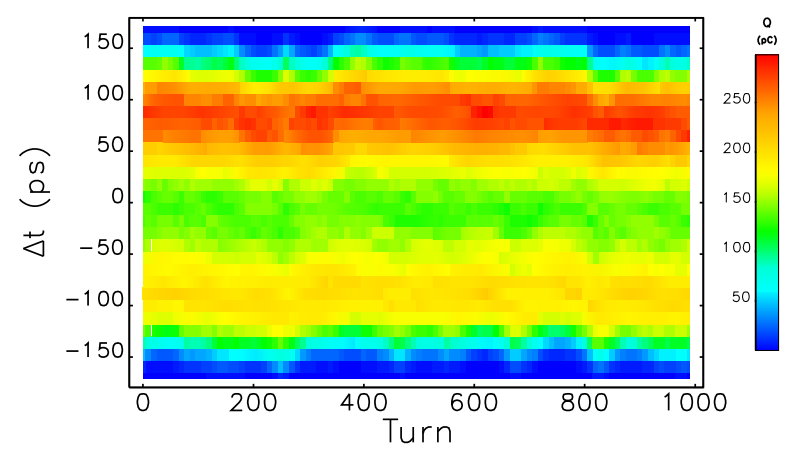

FIG. 16. Charge distribution over 1000 turns from 30-slice analysis for guard bunch 26 of a fill with twelve 2-bunch gaps. bunch 22 (normal charge), bunch 23 (double charge) and 26 (double charge) over 1000 turns (sampled every 10th turn) for the case with 12 gaps. Because of the presence of the short-range impedance and because the bunches are overstretched in order to increase the Touschek lifetime, the time distributions are not symmetric, but have two current peaks of about twice the central current. In most cases, the trailing peak is slightly higher than the leading peak. In the guard bunch before the gap, this is reversed, which is associated with a phase difference of $-10^{\circ}$ (at $1408 \mathrm{MHz}$ ) in the BLC seen by that bunch compared to the others; there is also a more modest $\left(-2.3^{\circ}\right.$ at $\left.352 \mathrm{MHz}\right)$ difference in the phase of the main cavities. There are also small voltage differences for this bunch, but it appears that the phase differences due to heavier single-pass beam loading are the main driver for the difference in the longitudinal distribution. With that exception, the bunch profiles are all very similar, which demonstrates that the guard bunch scheme controls rf transients fairly well.

For comparison, if uncompensated gaps are used, there is a $23^{\circ}$ linear phase variation for the harmonic cavity along the bunch train, accompanied by a $5^{\circ}$ linear phase variation for the main cavities. This introduces continuous variation in the bunch distribution from along the train. Figures 17 and 18 compare the phases with and without compensating bunches for the main and harmonic cavities in the 12-gap case. The voltage variation is modest regardless of whether or not compensated gaps are employed.

Computation of the Touschek lifetime requires the s-dependent momentum acceptance or "local" momentum acceptance (LMA) [38,39]. As part of the evaluation process for the APS-U lattice design, it is routine to compute the LMA for 100 cases of random errors and corrections [40]. To speed the computation of the lifetimes and provide a representative result, the s-dependent median LMA was used. Regardless of what is chosen for the LMA, the ratios of values of the lifetime will be unchanged.

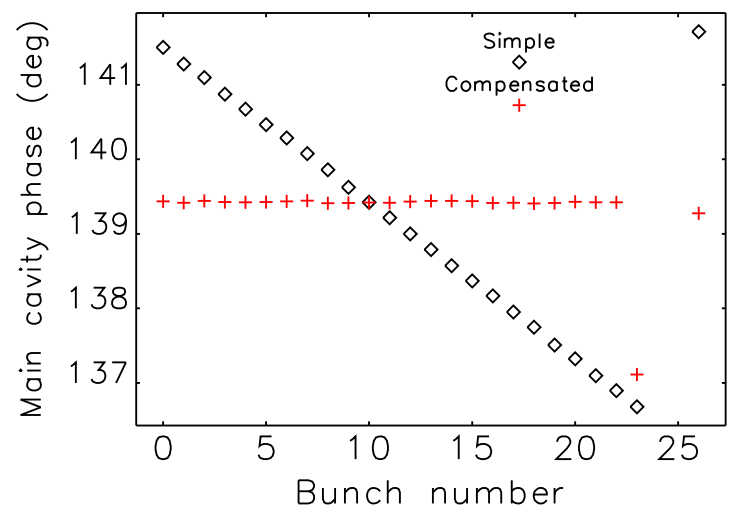

FIG. 17. Main-cavity phase of each unique bunch for the case of 12 simple gaps (uniform bunch charge) and 12 compensated gaps. 


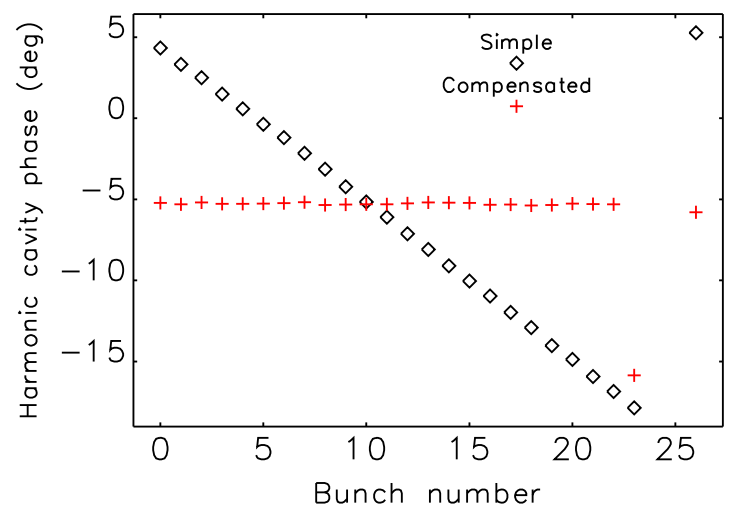

FIG. 18. Harmonic-cavity phase of each unique bunch for the case of 12 simple gaps (uniform bunch charge) and 12 compensated gaps.

Figure 19 shows the Touschek lifetimes for several patterns with various numbers of 2-bunch gaps. In each case, we see that the bulk of the bunches - those with the nominal charge-have nearly identical lifetime, whereas the double-charge bunches have lifetimes that are approximately $50 \%$ lower. This is very close to what one would anticipate if there was no significant difference in the longitudinal distributions for the two groups of bunches.

Because of their roughly two-fold lower Touschek lifetime combined with their two-fold higher charge, each guard bunch will create about four times as many particle losses as an ordinary bunch. However, each guard bunch in effect replaces two ordinary bunches, so the impact on total loss rate is reduced. Assuming that the loss rate $\frac{d N_{1}}{d t}$ for a bunch depends only on the bunch charge, it is expected that the total loss rate will be

$$
\frac{d N}{d t}=324 \frac{d N_{1}}{d t}\left(1+\frac{g}{81}\right)
$$

where $g$ is the number of 2-bunch gaps. Figure 20 shows the results from simulations, along with the expectation from Eq. (5) and a linear fit. The fit gives 1/90 for the coefficient of $g$, within $10 \%$ of the expected value.

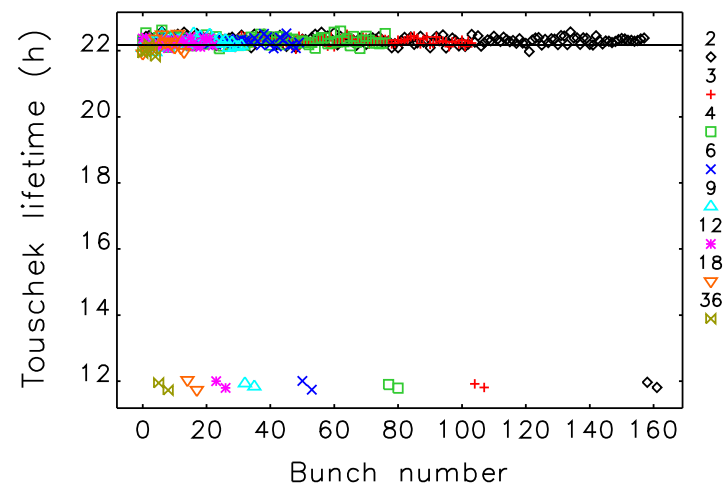

FIG. 19. Touschek lifetime for unique bunches for fill patterns with various numbers of two-bunch gaps in a 324-bunch pattern. The solid line shows the lifetime for a uniform 324-bunch fill.

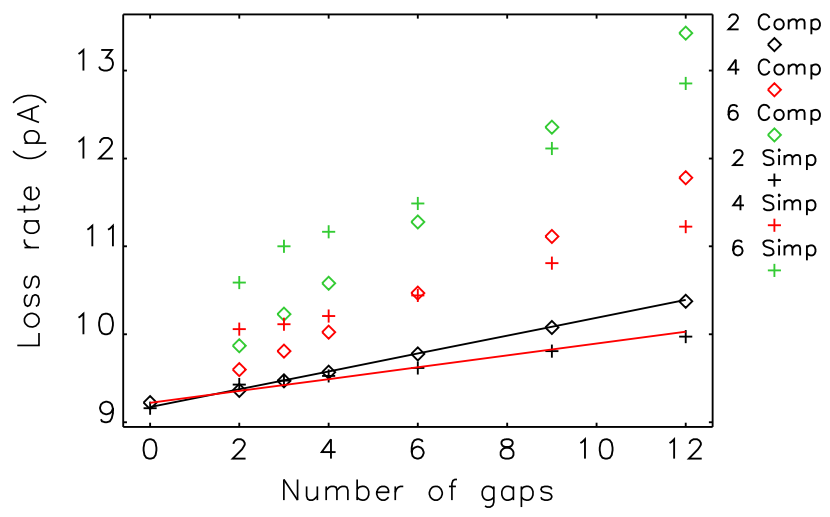

FIG. 20. Loss rate due to Touschek scattering as a function of the number of gaps in a 324-bunch pattern, for simple and compensated gaps, for various gap lengths. The solid lines show linear fits to the data for two-bunch gaps.

The smaller slope is expected because the guard bunches are longer than the normal bunches and have a lesspronounced variation of intensity along the bunch (see Figs. 13-16), because of a combination of potential well distortion and modest microwave instability. This results in their having about $8 \%$ longer lifetime than naively expected based on the charge alone.

Also shown for comparison in Fig. 20 are the loss rates for longer compensated gaps, along with those for simple (uncompensated) gaps. (In all the compensated-gap cases, the total number of guard bunches is equal to the number of bunches removed from the gaps, with each guard bunch having twice the charge of a normal bunch, so that the total charge in the beam is fixed. Similarly, in the uncompensated case the total charge in the beam is fixed, but all bunches have identical charge.) If two-bunch gaps are used, then for small numbers of gaps, there is little difference between the two methods of making gaps. For longer gaps, the compensated gap method shows a clear advantage when the number of gaps is relatively small, which it loses as the number of gaps increases. This is evidently due to the relatively modest variation in lifetime across the main part of the train with the simple gap method, coupled with the significantly higher loss rates from the guard bunches. Figure 21 illustrates this point.

\section{B. Ion simulations with compensated gaps}

The ion simulation code described above has been modified to allow for the modeling of compensated gaps. In addition to the number of bunch trains and the size of the gap between trains, the user specifies the number of guard bunches and the charge in each guard bunch. The code then simulates a bunch train with the specified number of guard bunches before and after the gap. In our simulations we assume that the missing charge from each empty bunch is added to one guard bunch, so the guard bunches have double the nominal charge. 


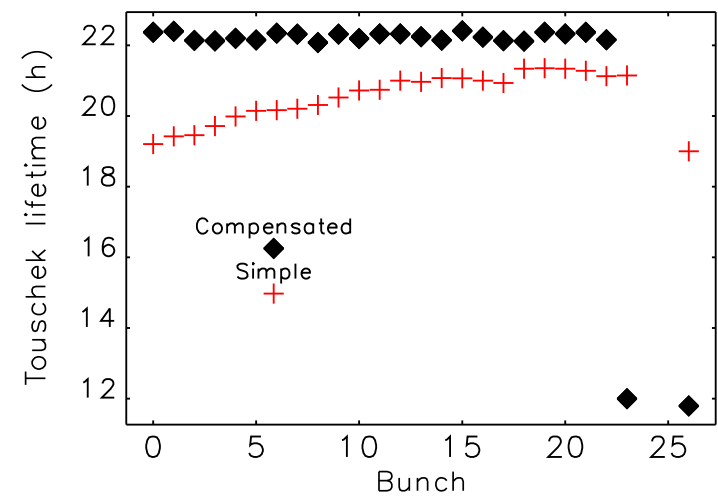

FIG. 21. Lifetime of unique bunches for the case of 12 twobunch gaps, comparing simple and compensated gaps.

Two parameters that can be varied in this model are the number of bunch trains and the size of the gap between trains. Increasing the number of missing bunches in the gap did not have an appreciable effect on the simulated instability growth rate. As discussed in the Appendix, a two-bunch gap with guard bunches narrows the trapping condition to the point that it is essentially irrelevant. This means that a twobunch gap will be effective at clearing out the ions, and using a larger gap will not provide much additional benefit.

Increasing the number of gaps has a more significant effect. Figure 22 shows the growth of the ion density during the first few turns (with 1000 Ah conditioning), for different numbers of train gaps. The density drops during the train gaps, and saturates at a relatively low value (compare to Fig. 6). Using more gaps leads to a lower saturation level.

This reduction in ion density is reflected in the instability amplitude (Fig. 23). With two gaps, the instability is nearly eliminated. The initial growth rate is reduced by almost two orders of magnitude, from $\sim 15 / \mathrm{ms}$ to $\sim 0.2 / \mathrm{ms}$. With 12 or more gaps there is no observable instability. Keeping in

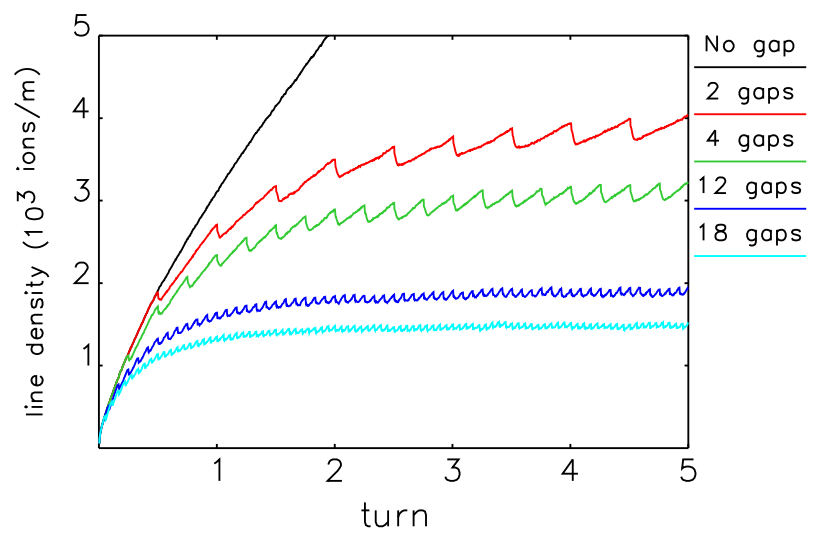

FIG. 22. Simulated ion density (averaged around the ring) for 50\% NEG coating, round beams, $200 \mathrm{~mA}, 1000 \mathrm{Ah}$ and 2-bunch gaps. Only the first few turns are shown.

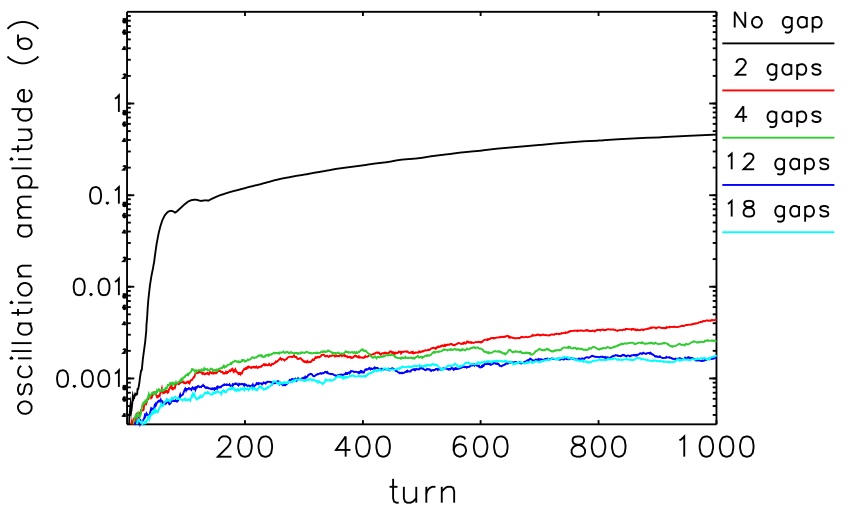

FIG. 23. Ion instability amplitude (in units of vertical beam size) for 50\% NEG coating, round beams, $200 \mathrm{~mA}, 1000 \mathrm{Ah}$, and 2-bunch gaps.

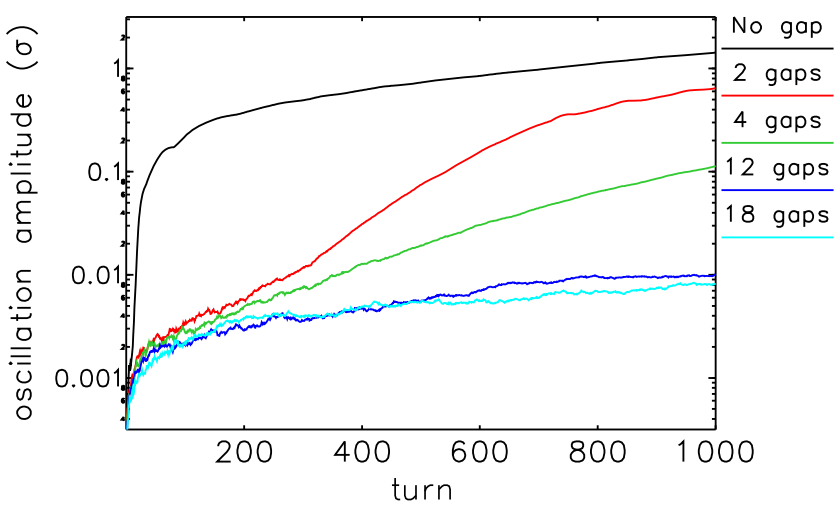

FIG. 24. Ion instability amplitude (in units of vertical beam size) for 50\% NEG coating, round beams, $200 \mathrm{~mA}, 100 \mathrm{Ah}$, and 2-bunch gaps.

mind that the expected vertical damping rate at the APS-U is $\sim 6-10 / \mathrm{ms}$, two gaps should be more than sufficient.

Figure 24 shows the train gap comparison for $100 \mathrm{Ah}$ beam conditioning. Using two train gaps reduces the initial growth rate from $\sim 33 / \mathrm{ms}$ to $\sim 0.9 / \mathrm{ms}$, still well below the expected vertical damping rate. Using more gaps further reduces the growth rate, down to $\sim 0.1 / \mathrm{ms}$ for 18 trains. In general, if two gaps proves to be insufficient (e.g., if the vacuum pressure is higher than expected), additional gaps can be used to further suppress the instability.

Of course, this scheme can also be deployed to avoid long-term trapping during commissioning. Using compensated gaps would allow us to use more bunches for a given total beam current (see Sec. III C). For example, running with two 2-bunch compensated gaps would allow for as many as 158 bunches at $25 \mathrm{~mA}$.

\section{SUMMARY}

Ion trapping is expected for 324 bunch mode in the APSUpgrade storage ring, and is likely to result in a vertical 
instability. This effect has been studied using a simulation code developed at SLAC, incorporating realistic pressure profiles generated by vacuum codes. The ions will be trapped in the multiplet sections, which were expected to have high pressure in the original vacuum design. The new design includes NEG coating in the multiplets, which will significantly reduce the severity of the instability, but not eliminate it. To further mitigate the ion instability, we plan to use train gaps, with double-charge guard bunches before and after the gap to reduce if transients. Simulations show that even two compensated gaps should be enough to reduce the growth rate below the rate of coherent damping and feedback, though more gaps can be used if necessary. We believe the compensated gap scheme would generally be effective at mitigating ion instability in other high charge, low emittance storage rings.

\section{ACKNOWLEDGMENTS}

We would like to thank L. Wang for providing his ion simulation code, J. Carter for providing APS-U pressure profiles, R. Lindberg for providing APS-U impedance data, and T. Berenc for providing the APS rf feedback model. This research was supported by the U.S. Department of Energy, Office of Science, under Contract No. DE-AC02$06 \mathrm{CH} 11357$.

\section{APPENDIX: DERIVING TRAPPING CRITERIA}

The ions receive a kick from each passing bunch, then drift in between bunches. In order for ions to be trapped, they must see focusing from the beam often enough that the beam current can be approximated as continuous. In other words, the bunch frequency must be much higher than the ion frequency.

This condition can be quantified by noting that the periodic focusing from the beam can be seen as analogous to the periodic focusing seen by the beam from quadrupole magnets. For ions of a given species at a given location in the lattice, we can define a periodic transfer matrix $\boldsymbol{M}_{\boldsymbol{p}}$, similar to the one turn transfer matrix for the beam. As in the beam case, the condition for ion stability is:

$$
\left|\operatorname{Tr} M_{p}\right| \leq 2
$$

where $\operatorname{Tr}$ is the trace operator.

\section{Continuous train}

For a continuous train with no gaps, the period is just a beam kick followed by a drift:

$$
M_{p, 0}=M_{k i c k} \times M_{d r i f t}
$$

For short bunches, the beam kick is effectively instantaneous, and the kick matrix has the same form as a thin quadrupole (except that it focuses in both planes).

$$
\boldsymbol{M}_{k i c k} \equiv\left(\begin{array}{cc}
1 & 0 \\
-a & 1
\end{array}\right)
$$

Here a is the kick from the beam:

$$
a \equiv \frac{2 N_{e} r_{p} Q}{A_{\text {ion }} \sigma_{y}\left(\sigma_{x}+\sigma_{y}\right)}
$$

where $A_{\text {ion }}$ is the atomic mass of the ion. Equation (A4) applies for the vertical plane; for the horizontal plane $\sigma_{x}$ and $\sigma_{y}$ are reversed.

The time between bunches is a drift:

$$
\boldsymbol{M}_{\text {drift }} \equiv\left(\begin{array}{cc}
1 & S_{b} \\
0 & 1
\end{array}\right)
$$

where $S_{b}$ is the bunch spacing. This makes:

$$
\boldsymbol{M}_{\boldsymbol{p}, \mathbf{0}} \equiv\left(\begin{array}{cc}
1 & S_{b} \\
-a & 1-a S_{b}
\end{array}\right)
$$

Plugging into Eq. (A1) gives the stability criteria:

$$
\left|2-a S_{b}\right| \leq 2
$$

Solving for $A_{\text {ion }}$ gives us the critical mass for ion trapping without gaps (Eq. (1)).

\section{Train gaps}

If there are gaps between bunch trains, the situation becomes a bit more complicated. Assuming there is trapping bunch-to-bunch, the train can be parameterized in terms of the total phase advance of the ions $\Phi \equiv$ $\omega_{i} n_{b} S_{b} / c$ (where $n_{b}$ is the number of bunches in the train).

$$
\boldsymbol{M}_{\text {train }} \equiv\left(\begin{array}{cc}
\cos (\Phi) & \frac{c}{\omega_{i}} \sin (\Phi) \\
-\frac{\omega_{i}}{c} \sin (\Phi) & \cos (\Phi)
\end{array}\right)
$$

where $\omega_{i}$ is given by Eq. (2). The train gap is another drift (of length $S_{g}$ ):

$$
\boldsymbol{M}_{\text {gap }} \equiv\left(\begin{array}{cc}
1 & S_{g} \\
0 & 1
\end{array}\right)
$$

The total period matrix is $\boldsymbol{M}_{\text {train }} \times \boldsymbol{M}_{\text {gap }}$ :

$\boldsymbol{M}_{p, t g} \equiv\left(\begin{array}{cc}\cos (\Phi) & S_{g} \cos (\Phi)+\frac{c}{\omega_{i}} \sin (\Phi) \\ -\frac{\omega_{i}}{c} \sin (\Phi) & \cos (\Phi)-\frac{\omega_{i} S_{g}}{c} \sin (\Phi)\end{array}\right)$

This gives a trapping criteria:

$$
\left|2 \cos (\Phi)-\frac{\omega_{i} S_{g}}{c} \sin (\Phi)\right| \leq 2
$$

Note that even with a train gap, there is a range of $\Phi$ where trapping still occurs (i.e., when the two terms in the absolute value are numerically close). Of course, this range becomes narrower as the size of the gap increases. 


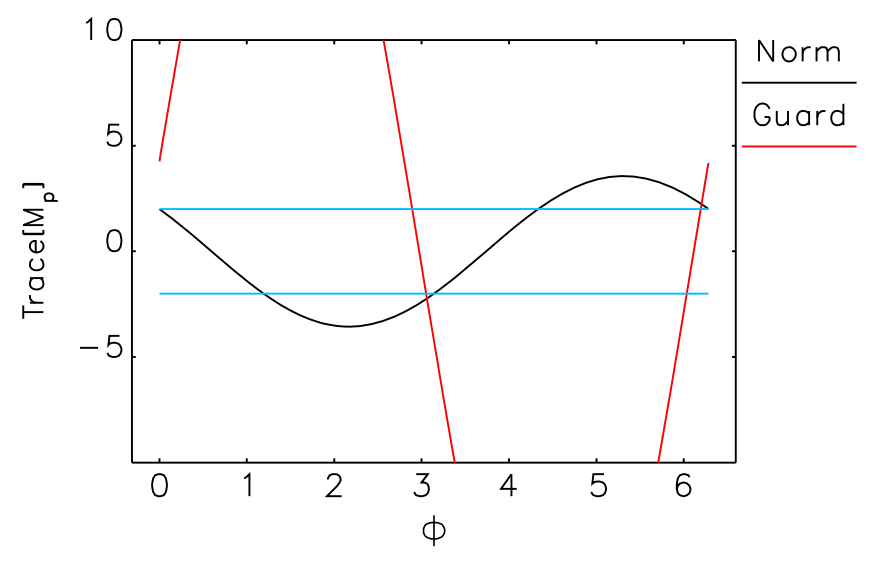

FIG. 25. Trace of the periodic matrix for a normal train with gaps, compared to a train with guard bunches. Trapping will occur when the absolute value of the trace is less then two.

\section{Compensated gaps}

To derive the trapping condition for the compensated gap scheme, the guard bunches must be counted separately from the rest of the train. For a fill pattern with one guard bunch at the start and end of each train, the periodic matrix becomes:

$$
\begin{aligned}
M_{p, c g}= & M_{\text {train }} \times M_{\text {guard }} \\
& \times M_{\text {gap }} \times M_{\text {guard }} \times M_{\text {drift }}
\end{aligned}
$$

Here $\boldsymbol{M}_{\text {train }}$ includes only the phase advance due to the normal charge bunches. In the APS-U case the guard bunches will have twice the normal charge, so:

$$
\boldsymbol{M}_{\text {guard }} \equiv\left(\begin{array}{cc}
1 & 0 \\
-2 a & 1
\end{array}\right)
$$

For a two bunch gap, $S_{g}=3 S_{b}$. Multiplying the matrices and taking the trace gives us the trapping condition with guard bunches:

$$
\begin{gathered}
\mid \frac{2\left[-2 a c^{2}+6 a^{2} c^{2} S_{b}-2 \omega_{i}^{2} S_{b}+3 a \omega_{i}^{2} S_{b}^{2}\right] \sin (\Phi)}{c \omega_{i}} \\
+2\left[1+2 a S_{b}\left(-4+3 a S_{b}\right)\right] \cos (\Phi) \mid \leq 2
\end{gathered}
$$

Figure 25 plots the trace of the periodic matrices with and without guard bunches (Eqs. (A11) and (A14), as a function of the phase advance along the train. The parameters are chosen for maximum trapping: $\mathrm{CO}_{2}$ $(\mathrm{A}=44)$ at the point in the APS-U lattice with minimum $\mathrm{A}_{\text {crit }}\left(\sigma_{x}=87 \mu \mathrm{m}, \sigma_{y}=21 \mu \mathrm{m}\right)$. Trapping will occur when the absolute value of the trace is less than two. For the case without guard bunches, there is a broad range of $\Phi$ where trapping can still occur. This range is much narrower for the case with guard bunches.
Note that there is an equivalent condition in the horizontal plane that must also be met. In addition, these trapping equations assume a linear focusing force independent of ion position. In reality, the focusing from the Gaussian beam starts to roll off around one beam $\sigma$, and decays after that. If the position of the ion in either plane is ever far from the beam centroid, the conditions are no longer valid. Therefore they should be understood as necessary but not sufficient conditions for trapping.

[1] Advanced photon source upgrade project preliminary design report, Technical Report No. APSU-2.01-RPT-002, 2017. https://www.aps.anl.gov/APS-Upgrade/Documents.

[2] M. Borland, Y. P. Sun, V. Sajaev, R. R. Lindberg, and T. Berenc, Lower emittance lattice for the advanced photon source upgrade using reverse bending magnets, in Proc. of NAPAC16, pp. 877-880, 2016, https://doi.org/10.18429/ JACoW-NAPAC2016-WEPOB01.

[3] L. Farvacque, N. Carmignani, J. Chavanne, A. Franchi, G. Le Bec, S.Liuzzo, B. Nash, T. Perron, and P. Raimondi, A low-emittance lattice for the ESRF, in Proceedings of the 25th Particle Accelerator Conference, PAC-2013, Pasadena, CA, 2013 (IEEE, New York, 2013), pp. 79-81.

[4] J. Delahaye and J. P. Potier, Reverse bending magnets in a combined-function lattice for the CLIC damping ring, in Proceedings of the 1989 Particle Accelerator Conference, Chicago, IL (IEEE, New York, 1989), pp. 1611-1613.

[5] A. Streun, The anti-bend cell for ultralow emittance storage ring lattices, Nucl. Instrum. Methods Phys. Res., Sect. A 737, 148 (2014).

[6] H. G. Hereward, Coherent instability due to electrons in a coasting proton beam, CERN Technical Report No. CERN-71-15, 1971.

[7] D. Villevald and S. Heifets, Ion trapping in the SLAC Bfactory high energy ring, SLAC Technical Report No. SLAC-TN-06-032, 1993.

[8] Y. Baconnier and G. Brianti, The stability of ions in bunched beam machines, CERN Technical Report No. CERN/SPS/80-2, 1980.

[9] C. Bernardini, G. F. Corazza, G. Di Giugno, G. Ghigo, J. Haissinski, P. Marin, R. Querzoli, and B. Touschek, Lifetime and Beam Size in a Storage Ring, Phys. Rev. Lett. 10, 407 (1963).

[10] A. Xiao and M. Borland, Intra-beam and Touschek scattering computations for beam with non-gaussian longitudinal distributions, in Proc. of IPAC 2015, pp. 559-561, 2015, https://doi.org/10.18429/JACoWIPAC2015-MOPMA012.

[11] L. Wang, Y. Cai, T. Raubenheimer, and H. Fukuma, Suppression of beam-ion instability in electron rings with multibunch train beam fillings, Phys. Rev. Accel. Beams 14, 084401 (2011).

[12] L. Wang, J. Safranek, Y. Cai, J. Corbett, R. O. Hettel, T. O. Raubenheimer, J. Schmerge, and J. Sebek, Beam ion instability: Measurement, analysis, and simulation, Phys. Rev. Accel. Beams 16, 104402 (2013). 
[13] M. Bassetti and G. Erskine, Closed expression for the electrical field of a two-dimensional Gaussian charge, CERN Technical Report No. CERN-ISR-TH-80-06, 1983.

[14] J. Calvey, K. Harkay, and C. Yao. Ion effects in the aps particle accumulator ring. In NAPAC16, 2016. THPOA14, https://doi.org/10.18429/JACoW-NAPAC2016-THPOA14.

[15] M. Ady and R. Kersevan, Introduction to the latest version of test particle Monte Carlo code MOLfLow, in Proc. IPAC 2014 (IEEE, NewYork, 2014), p. 2348, http://accelconf .web.cern.ch/AccelConf/IPAC2014/papers/wepme038 .pdf.

[16] R. Kersevan, SYNRAD, a Monte Carlo synchrotron radiation ray tracing program, in Proceedings of the 15th Particle Accelerator Conference, PAC-1993, Washington, DC, 1993 (IEEE, New York, 1993), p. 3848. http://accelconf .web.cern.ch/AccelConf/p93/PDF/PAC1993_3848.PDF.

[17] J. A. Carter, Benchmarking and Calibration of Monte Carlo Vacuum Simulations with SynRad and MolFlow+, In Proc. of International Particle Accelerator Conference (IPAC'16), Busan, Korea, May 8-13, 2016 (JACoW, Geneva, Switzerland, June 2016), pp. 3695-3697, https://doi.org/10.18429/JACoW-IPAC2016-THPMY018.

[18] J. A. Carter, B. Billett, B. Brajuskovic, M. A. Lale, A. McElderry, J. R. Noonan, M. M. O’Neill, K. Wakefield, D. R. Walters, G. E. Wiemerslage, and J. Zientek. Progress on the final design of the APS-Upgrade storage ring vacuum system, in Proc. 10th Mechanical Engineering Design of Synchrotron Radiation Equipment and Instrumentation (MEDSI'18), Paris, France, 25-29 June 2018 (JACoW Publishing, Geneva, Switzerland, 2018), pp. 30-32, https:// doi.org/10.18429/JACoW-MEDSI2018-TUPH04.

[19] M. Ady, P. Chiggiato, R. Kersevan, Y. Tanimoto, and T. Honda, Photodesorption and electron yield measurements of thin film coatings for future accelerators, in Proc. of IPAC2015, pp. 3123-3126, 2015, https://doi.org/ 10.18429/JACoW-IPAC2015-WEPHA011.

[20] G. V. Stupakov and A. W. Chao, Study of beam decoherence in the presence of head-tail instability using a two-particle model, in Proceedings of the Particle Accelerator Conference, Dallas, TX, 1995 (IEEE, New York, 1995), pp. 3288-3290.

[21] M. G. Minty, A. W. Chao, and W. L. Spence, Emittance growth due to decoherence and wakefields, in Proceedings of the Particle Accelerator Conference, Dallas, TX, 1995 (IEEE, New York, 1995), pp. 3037-3039.

[22] C. Yao, E. Norum, and N. DiMonte, An FPGA-based bunch-to-bunch feedback system at the advanced photon source, in Proceedings of the 22nd Particle Accelerator Conference, PAC-2007, Albuquerque, NM (IEEE, New York, 2007), p. 440, http://accelconf.web.cern.ch/ AccelConf/p07/PAPERS/MOPAN116.PDF.

[23] T. O. Raubenheimer and F. Zimmermann, Fast beam-ion instability. I. Linear theory and simulations, Phys. Rev. E 52, 5487 (1995).

[24] G. V. Stupakov, T. O. Raubenheimer, and F. Zimmermann, Fast beam-ion instability. II. Effect of ion decoherence, Phys. Rev. E 52, 5499 (1995).

[25] J. Byrd, A. Chao, S. Heifets, M. Minty, T. O. Raubenheimer, J. Seeman, G. Stupakov, J. Thomson, and F. Zimmermann,
First Observations of a "Fast Beam-Ion Instability", Phys. Rev. Lett. 79, 79 (1997).

[26] K. L. F. Bane, K. Kubo, and P. B. Wilson, Compensating the unequal bunch spacings in the NLC damping rings, in Proc. EPAC 1996, pp. 1111-1113, 1996, http://accelconf .web.cern.ch/AccelConf/e96/PAPERS/WEPG/WEP113G .PDF.

[27] J. M. Byrd, S. De Santis, J. Jacob, and V. Serriere, Transient beam loading effects in harmonic rf systems for light sources, Phys. Rev. Accel. Beams 5, 092001 (2002).

[28] H. Wang, R. A. Rimmer, S. Wang, D. Teytelman, Y. Zhang, J. Yue, J. Dai, J. Xing, and Q. Qin, Transient beam loading due to the bunch train gap and its compensation experiments at BEPC-II and ALS, in Proc. IPAC 2018, pp. 390-393, 2018, https://doi.org/10.18429/JACoW-IPAC2018-MOPMK017.

[29] Y. Wang and M. Borland, Implementation and performance of parallelized ELEGANT, in Proceedings of the 22nd Particle Accelerator Conference, PAC-2007, Albuquerque, NM (IEEE, New York, 2007), pp. 3444-3446.

[30] Y. Wang, M. Borland, H. Shang, and R. Soliday, Recent progress on parallel elegant, in ICAP 2009, p. 355, 2009, http://accelconf.web.cern.ch/AccelConf/ICAP2009/papers/ thpsc054.pdf.

[31] M. Borland, elegant: A flexible sdds-compliant code for accelerator simulation, Advanced Photon Source Technical Report No. LS-287, 2000.

[32] M. Borland, T. Berenc, L. Emery, and R. Lindberg, Simultaneous simulation of multi-particle and multi-bunch collective effects for the APS ultra-low emittance upgrade, in Proceedings of the 2015 International Computational Accelerator Physics Conference, pp. 61-65, 2015, http:// accelconf.web.cern.ch/AccelConf/ICAP2015/papers/webji1 .pdf.

[33] M. Borland, R. Lindberg, and A. Xiao, Improvements in modeling of collective effects in ELEGANT, in Proc. of IPAC 2015, p. 549, 2015, https://doi.org/10.18429/ JACoW-IPAC2015-MOPMA009.

[34] R. R. Lindberg and A. Blednykh, Modeling of impedance effects for the APS-MBA Upgrade, in Proceedings of IPAC15, pp. 1825-1827, 2015, https://doi.org/10.18429/ JACoW-IPAC2015-TUPJE078.

[35] R. R. Lindberg and A. Blednykh, Instability thresholds for the Advanced Photon Source multi-bend achromat upgrade, in Proceedings of IPAC15, pp. 1822-1824, 2015, https://doi.org/10.18429/JACoW-IPAC2015-TUPJE077.

[36] T. Berenc and M. Borland, Modeling rf feedback in ELEGANT for bunch-lengthening studies for the Advanced Photon Source Upgrade, In Proc. of IPAC 2015, p. 540, 2015, https://doi.org/10.18429/JACoW-IPAC2015-MOPMA006.

[37] M. P. Kelly, A. Barcikowski, J. Carwardine, Z. A. Conway, D. Horan, M. Kedzie, S. H. Kim, P. N. Ostroumov, J. Rathke, T. Schultheiss, and G. J. Waldschmidt, Superconducting harmonic cavity for the Advanced Photon Source upgrade, in Proceedings of IPAC15, pp. 3267-3269, 2015, https://doi.org/10.18429/JACoW-IPAC2015-WEPTY008.

[38] C. Steier, D. Robin, L. Nadolski, W. Decking, Y. Wu, and J. Laskar, Measuring and optimizing the momentum aperture in a particle accelerator, Phys. Rev. E 65, 056506 (2002). 
[39] M. Belgroune, P. Brunelle, A. Nadji, and L. Nadolski, Refined tracking procedure for the SOLEIL energy acceptance calculation, in Proceedings of the 25th Particle Accelerator Conference, PAC-2013, Pasadena, CA, 2013 (IEEE, New York, 2013), pp. 896-898.
[40] V. Sajaev and M. Borland, Commissioning simulations for the APS Upgrade Lattice, in Proceedings of IPAC15, pp. 553-555, 2015, https://doi.org/10.18429/JACoWIPAC2015-MOPMA010. 